\title{
CONFLICTOS ÉTICOS DETECTADOS POR PSICÓLOGOS/AS DE LA ATENCIÓN PRIMARIA DE SALUD
}

\begin{abstract}
Manuel Pérez Ayala ${ }^{1}$
Resumen Objetivo: Identificar la detección de conflictos éticos por parte de los psicólogos/as en el contexto de la atención primaria de salud (APS); determinar su compresión y analizar la descripción de cada uno. Diseño la investigación: Estudio cualitativo, tipo exploratorio-descriptivo. Participantes: 25 psicólogas/os de un universo total de 28 profesionales. Método: El instrumento de recolección de información fue la entrevista semiestructurada, y la técnica de análisis de resultados el análisis narrativo de contenido. Resultados: Los informantes detectan un total de treinta y ocho conflictos éticos claramente diferenciados, argumentando a catorce de ellos como críticos. La categorización permitió diferenciar tres categorías distintas desde las que se establecen y representan: a) atención a consultantes; b) relación entre colegas y con otros profesionales de salud, y c) contexto de trabajo en salud pública. Conclusiones: El estudio permitió acceder a las problemáticas percibidas por los informantes, coincidiendo en que varios de estos conflictos han permanecido naturalizados y normalizados en el tiempo. Surge la necesidad de revisar y visibilizar los cuestionamientos identificados para que establezcan los mínimos necesarios que permitan ser garantes de intervenciones de calidad y excelencia requerida en la salud mental del nivel primario de atención.
\end{abstract}

Palabras clave: psicólogos, ética, conflictos éticos, atención primaria de salud

\section{Ethical conflicts detected by psychologists of Primary Health Care}

\begin{abstract}
Objectives: To identify the detection of ethical conflicts by psychologists in the context of PHC, determine their understanding and analyze the description of each one. Design: Qualitative study, exploratory-descriptive type. Participants: 25 psychologists, from a total universe of 28 professionals. Method: The data collection instrument was the semi-structured interview and the results analysis technique was the narrative analysis of content. Results: The informants detected a total of thirty-eight clearly differentiated ethical conflicts, arguing fourteen of them as critics. The categorization allowed differentiating three different categories from where they are established and represent: a) attention to consultants; b) relationship between colleagues and with other health professionals; and c) work context in public health. Conclusions The study allowed access to the problems perceived by the informants, agreeing that several of these conflicts have remained naturalized and normalized over time. The need arises to review and make visible the identified questions in order to establish the minimums necessary to be guarantors of quality interventions and excellence required in the mental health of the primary level of care.
\end{abstract}

Keywords: psychologists, ethics, ethical conflicts, primary health care

\section{Conflitos éticos detectados por psicólogos/as da atenção primária de saúde}

Resumo: Objetivo: Identificar a deteç̧ão de conflitos éticos por parte dos Psicólogos/as no contexto da APS, determinar sua compreensão e analisar a descrição de cada um. Desenho da pesquisa: Estudo qualitativo, tipo exploratório-descritivo. Participantes: 25 Psicólogos/as, de um universo total de 28 profissionais. Método: O instrumento de coleta de informaçóes foi uma entrevista semiestruturada e a técnica de análise de resultados, a análise narrativa de conteúdo. Resultados: Os informantes detectam um total de trinta e oito conflitos éticos claramente diferenciados, considerando quatorze deles como críticos. A categorização permitiu diferenciar três categorias distintas de onde eles estão estabelecidos e representam: a) atenção a pessoa em consulta; b) relação entre colegas e com outros profissionais da saúde; e c) contexto de trabalho em saúde pública. Conclusóes: $\mathrm{O}$ estudo permitiu acessar as problemáticas percebidas pelos informantes, coincidindo em que vários destes conflitos permaneceram naturalizados e padronizados no tempo. Surge a necessidade de revisar e visibilizar os questionamentos identificados para que se estabeleçam os padróes* mínimos necessários que permitam ser garantidores de intervençóes de qualidade e excelência requeridas na saúde mental do nível primário de atenção.

Palavras chave: psicólogos, ética, conflitos éticos, atenção primária de saúde

\footnotetext{
${ }^{1}$ Escuela de Psicología, Universidad Autónoma de Chile, Chile Correspondencia: mperezayala@gmail.com
} 


\section{Introducción}

En la atención primaria en salud (APS) se debe resolver aproximadamente el $85 \%$ de los problemas de salud de la población(1). Su objetivo en sí no es tanto intervenir las enfermedades concretas como sí lo es el manejo integral de la salud de los usuarios y usuarias, a quienes en todo su ciclo vital no se les ve como sujetos aislados, desligados de sus contextos sociales y humanos, sino como incorporados en sus estructuras familiares y comunitarias(2).

Con el énfasis promocional y preventivo de sus estrategias, en la APS el pilar es el equipo de salud, centrado en mejorar la accesibilidad de prestaciones de salud a los usuarios/usuarias y sus familias(3), equipo que progresivamente debe capacitarse en diversas áreas para alcanzar intervenciones más integrales y de alta calidad.

En esa línea, es una tendencia mayoritaria y a escala global que en las organizaciones de salud se estimule un creciente y general interés sobre la importancia del accionar ético de las personas que ahí laboran(4). Un comportamiento encuadrado en la ética se basa en impulsar un ambiente en el que prime el profesionalismo, en la gestión orientada a la calidad total del servicio y en el diálogo con el contexto y especificación de su marco de responsabilidad social; todas acciones dirigidas hacia la promoción de la calidad y excelencia.

La práctica del trabajo en salud que contempla atención de personas está necesariamente asociada a conflictos de distinta índole: personales, profesionales y éticos(5). En la APS confluyen una serie de cuestiones éticas que han recibido una atención insuficiente debido a que no son percibidas como tales, y que requieren una especial consideración. Reviste especial importancia que los profesionales, técnicos y trabajadores en general declaren y compartan principios y valores, e interioricen que la ética en la atención hacia aquellos que reciben el servicio adquiere un significado especial en el comportamiento de la persona que lo brinda(6), desde los abocados al trato directo hasta los directivos de la institución que los oferta. En este contexto, el profesional de salud tiene que aprender el correcto procedimiento de su práctica no solo de hechos clínicos o comunitarios, sino también de los valores y los deberes que les son inherentes(7).

Si bien la exigencia en este nivel es múltiple, existe relativo acuerdo en cuanto a delinear las funciones y rol específicos de los psicólogos que ahí se desempeñan. Sus competencias profesionales se relacionan con capacidades para responder a contextos individuales, grupales y comunitarios, con énfasis en la promoción y la prevención, trabajar cooperativamente con otros miembros del equipo y apoyar el desarrollo de instituciones saludables comenzando por el propio establecimiento, con una comprensión amplia del proceso de salud/ enfermedad(8).

De sus particulares requerimientos, la dimensión ética no es excepción(9), adquiriendo particular relevancia dado el tipo de demandas a las que se debe dar cumplimiento. Se hace necesario indagar en la existencia y/o detección por parte de los profesionales sobre conflictos éticos, primero para su concepción y comprensión, y luego para su análisis y evaluación de estrategias resolutorias. El levantamiento y socialización de información como ésta ayudaría a estandarizar y sistematizar formas concretas de abordaje, que permitan además perfeccionar cada práctica.

El objetivo principal de este estudio fue detectar conflictos éticos en el ejercicio profesional de psicólogas y psicólogos en el contexto de APS. Entre los objetivos específicos se encuentran determinar la comprensión y analizar los conflictos éticos descritos por los entrevistados, estableciendo un orden prioritario de ellos que permita además argumentar la justificación de los que consideren críticos, entendidos estos como los que necesitan una resolución inmediata o al menos diligente por sobre los otros advertidos, debido a factores como periodo de extensión desde su detección, magnitud, implicancias y/o alcances.

\section{Método}

Se realizó un estudio cualitativo, de tipo exploratorio-descriptivo, durante el segundo semestre de 2017 y el primero de 2018. El protocolo de investigación fue revisado y aprobado por el Comité de Ética Científica del Servicio de Salud del Maule, con fecha 7 de julio de 2017, y por el Comité de 
Relaciones Asistenciales Docentes de la Dirección Comunal de Salud de Talca, con fecha 2 de agosto de 2017.

Participaron psicólogos y psicólogas que desempeñan labores en APS de la comuna de Talca, Región del Maule. Abarcó a los diez establecimientos que conforman la red de APS: siete CESFAM (Centros de Salud Familiar), dos CECOSF (Centros Comunitarios de Salud Familiar), y una Posta de Salud Rural.

La información fue recopilada a través de una entrevista semiestructurada, la cual fue grabada en formato audio con la autorización de cada entrevistado/a, según consignaba uno de los puntos del proceso de consentimiento informado. Las grabaciones quedaron en custodia del autor, con el acuerdo de ser borradas doce meses después del fin de la investigación, que contempla la devolución de información al estamento de psicólogas/ os del Departamento de Salud comunal de Talca, mediante formato a convenir.

El guión temático fue específicamente confeccionado para esta investigación, y contó con la evaluación de dos pares expertos, más una ronda de tres entrevistas piloto antes de su diseño definitivo. Los principales ejes temáticos consultados fueron: a) qué se entendía por ética; b) detección o no de conflictos éticos en el ejercicio profesional; c) identificar conflicto, cuál y por qué era considerado crítico; d) estrategias probadas para abordar tales conflictos, y e) estrategias propuestas para abordar tales conflictos.

Todas las entrevistas fueron efectuadas en los propios establecimientos de trabajo de cada

\begin{tabular}{|c|c|c|c|c|c|c|}
\hline Informante & Sexo & Edad & $\begin{array}{c}\text { Ańos } \\
\text { desde } \\
\text { egreso }\end{array}$ & $\begin{array}{c}\text { Años de trabajo en } \\
\text { APS }\end{array}$ & $\begin{array}{l}\text { Años de trabajo en el } \\
\text { centro de salud }\end{array}$ & $\begin{array}{c}\text { Carga horaria } \\
\text { semanal }\end{array}$ \\
\hline 1 & $\mathbf{M}$ & 36 & 9 & 9 & 9 & 44 hrs \\
\hline 2 & $\mathbf{F}$ & 50 & 25 & 20 & 20 & $44 \mathrm{hrs}$ \\
\hline 3 & $\mathbf{F}$ & 40 & 13 & 13 & 1 & 44 hrs \\
\hline 4 & $\mathbf{M}$ & 49 & 12 & 9 & 3 & 44 hrs \\
\hline 5 & $\mathbf{M}$ & 33 & 9 & 6 & 2 & $44 \mathrm{hrs}$ \\
\hline 6 & $\mathbf{M}$ & 38 & 11 & 11 & 10 & 44 hrs \\
\hline 7 & $\mathbf{F}$ & 37 & 12 & 11 & 11 & 44 hrs \\
\hline 8 & $\mathbf{F}$ & 33 & 9 & 5 & 5 & 44 hrs \\
\hline 9 & $\mathbf{F}$ & 31 & 5 & 2 & 2 & 44 hrs \\
\hline 10 & $\mathbf{M}$ & 28 & 6 & 6 & 6 & $44 \mathrm{hrs}$ \\
\hline 11 & $\mathbf{F}$ & 32 & 7 & 7 & 7 & 44 hrs \\
\hline 12 & $\mathbf{F}$ & 29 & 6 & 2 & 2 & 44 hrs \\
\hline 13 & $\mathbf{M}$ & 43 & 20 & 19 & 10 & $44 \mathrm{hrs}$ \\
\hline 14 & $\mathbf{F}$ & 45 & 11 & 11 & 11 & 44 hrs \\
\hline 15 & $\mathbf{F}$ & 45 & 11 & 11 & 11 & 44 hrs \\
\hline 16 & $\mathbf{F}$ & 33 & 9 & 9 & 6 & $44 \mathrm{hrs}$ \\
\hline 17 & $\mathbf{F}$ & 31 & 6 & 6 & 6 & 44 hrs \\
\hline 18 & $\mathbf{F}$ & 34 & 9 & 4 & 3 & $44 \mathrm{hrs}$ \\
\hline 19 & $\mathbf{M}$ & 47 & 8 & 5 & 4 & 44 hrs \\
\hline 20 & $\mathbf{F}$ & 35 & 7 & 7 & 1 & 44 hrs \\
\hline 21 & $\mathbf{M}$ & 28 & 4 & 3 & 3 & $44 \mathrm{hrs}$ \\
\hline 22 & $\mathbf{F}$ & 45 & 11 & 11 & 9 & $44 \mathrm{hrs}$ \\
\hline 23 & $\mathbf{M}$ & 42 & 8 & 6 & 6 & 44 hrs \\
\hline 24 & $F$ & 34 & 8 & 7 & 7 & 44 hrs \\
\hline 25 & $F$ & 29 & 4 & 4 & 4 & $44 \mathrm{hrs}$ \\
\hline
\end{tabular}

Tabla 1: Caracterización sociodemográfica y profesional de los informantes. 
entrevistado/a, en días y horarios previamente acordados, durante el periodo septiembre 2017 y enero 2018. Tuvieron un rango de duración de 23 minutos (Informante 4), la más breve, y de 47 minutos (Informante 7), la más extensa. La técnica de análisis de resultados fue el análisis narrativo de contenido, mediante la construcción de categorías conceptuales relacionadas con la temática eje.

\section{Resultados}

De un universo de 28 profesionales totales que laboran en APS, 25 accedieron a participar. De éstos, 16 fueron mujeres y 9 hombres, siendo el promedio de edad 37 años. Respecto del promedio de años desde su egreso, este es de 9,6 años; el de trabajo en APS es de 8,1 años y el de trabajo en el Centro de Salud actual al momento de la entrevista es de 6,3 años. El detalle se puede apreciar en la tabla 1 .

\section{Conflictos éticos detectados}

Atención a compañeros de labores y/o a sus familiares

Extenso periodo intercontrol de usuarios/as

Sobredemanda de funciones como impedimento de calidad en cometidos

Obligatoriedad de denunciar y su relación costo/efectividad

Limitada resolutividad de nivel de atención

Infraestructura deficiente e insuficiente para realizar distintos abordajes

Percepción que intervenciones no solucionan problemáticas reales

Contrariedad de perspectivas con instituciones derivantes o de derivación

Obligatoriedad de atención a usuarios/as con actitudes de hostigamiento/acoso

Criterios disímiles ante contrerreferencias desde nivel secundario

Ocasional trato irrespetuoso de administrativos a usuarios/as

Discrepancia en atención a usuarios con determinado credo religioso

Significación de la violencia normalizada en parte de la población control

Salud Mental como programa relegado en APS

Manejo de confidencialidad con o sin información cruzada

Aclaración y cumplimiento del rol del psicólogo/a y sus funciones

Atención a personas con comportamiento erotizado

Atención personas de la diversidad sexual sin capacitación correspondiente

Reconocimiento de los límites en la intervención de salud mental

Aceptar obsequios o similares por atención que es gratuita

Solicitud de atención en casos especiales, no respetando orden establecido

Atención a hombres que han perpetrado hechos de abuso

Atención a solo un integrante en contexto de terapia de pareja

Solicitud de informes/certificados que tergiversen o falseen información

Implicancias de la estigmatización del usuario/as de salud mental

Acceso a información especialmente sensible de usuarios/as

Develación de información privada en reuniones profesionales

Resguardo de información especialmente sensible en ficha electrónica

Respeto a normativas de coordinación, en trato con otros funcionarios/as

Desacuerdo ante intervenciones con funcionarios ajenos al área psicosocial

Faltas de respeto y desautorizaciones entre pares funcionarios

Disenso con equipo profesional sobre tratamientos idóneos

Apresuradas derivaciones a otros tratamientos o nivel secundario

Falta de reuniones para revisión de casos clínicos

Postura profesional ante situaciones de negligencia dentro de la institución

Excesivas atribuciones del médico, sin formación/competencias necesarias

Desmesurada medicalización a usuarios/as de salud mental

Nivel precario competencias clínicas, considerando perfil riesgoso de usuarios

\section{Total menciones Total menciones como críticos}

11

5

3

3

2

2

1

1

1

1

1

1

1

1

5

4

2

2

1

1

1

1

1

1

1

1

1

1

1

1

1

1

1

1

1

1

1

1

Tabla 2: Totalidad de conflictos éticos detectados por los entrevistados. Se muestra división de los descritos y los priorizados como críticos, con las respectivas veces (número) que fueron espontáneamente mencionados. 
Los profesionales entrevistados identificaron un total de treinta y ocho conflictos éticos distintos, justificando y argumentando a catorce de ellos como críticos (tabla 2). Los informantes enten- dían y expresaban a los conflictos éticos en su ejercicio a través de aspectos como: “ (...) contraposiciones morales que alteran la aplicación profesional”, Informante 1 - Respuesta 1; “ (...) situacio-

\section{CATEGORÍA}

\section{ATENCIÓN A} CONSULTANTES

\section{RELACIÓN}

ENTRE COLEGAS

Y CON OTROS

PROFESIONALES DE

LA SALUD

\section{CONTEXTO DE TRABAJO EN SALUD PÚBLICA}

\section{SUBCATEGORÍAS \\ Encuadre de las intervenciones}

Juicio, congruencia y sentido del deber profesional

\section{Gestión de la confidencialidad}

\section{CONFLICTOS ÉTICOS DETECTADOS}

Reconocimiento de los límites en la intervención de salud mental Aceptar obsequios o similares por atención que es gratuita Solicitud de atención en casos especiales, no respetando orden establecido

Obligatoriedad de atención a usuarios/as con actitudes hostigamientolacoso Discrepancia en atención a usuarios con determinado credo religioso Atención a hombres que han perpetrado hechos de abuso Atención a sólo un integrante en contexto de terapia de pareja Atención a personas con comportamiento erotizado Solicitud de informes/certificados que tergiversen o falseen información Implicancias de la estigmatización del usuario/as de Salud Mental

Obligatoriedad de denunciar y su relación costolefectividad Manejo de confidencialidad con o sin información cruzada Acceso a información especialmente sensible de usuarios/as Develación de información privada en reuniones profesionales Resguardo de información especialmente sensible en ficha electrónica

Impacto de distintos abordajes

Percepción que intervenciones no solucionan problemáticas reales Limitada resolutividad de nivel de atención

\section{Establecimiento y cumplimiento de límites Discrepancias respecto a
tratamientos y tratos} Atención a compañeros de labores y/o a sus familiares Respeto a normativas en coordinación, en trato con Contrariedad de perspectivas con instituciones derivantes o de derivación Ocasional trato irrespetuoso de administrativos a usuarios/as Desacuerdo ante intervenciones con funcionarios ajenos al área psicosocial

Faltas de respeto y desautorizaciones entre pares funcionarios Disenso con equipo profesional sobre tratamientos idóneos

Coordinación general de actividades

Criterios disimiles ante contrerreferencias desde nivel secundario Apresuradas derivaciones a otros tratamientos o nivel secundario Falta de reuniones para revisión de casos clínicos

Definición, apropiación y ejecución de rol profesional

Significación de la violencia normalizada en parte de la población control Salud Mental como programa relegado en APS

Aclaración y cumplimiento del rol del psicólogo/a y sus funciones Postura profesional ante situaciones de negligencia dentro de la institución

\author{
Nivel y tipo de recursos \\ existentes $\mathrm{v} / \mathrm{s}$ necesarios
}

Exigencias formativas $y$ de capacitación

\section{Extenso periodo intercontrol de usuarios/as}

Infraestructura deficiente e insuficiente para realizar distintos abordajes Sobredemanda de funciones como impedimento de calidad en cometidos

Atención personas de la diversidad sexual sin capacitación correspondiente Excesivas atribuciones del Médico, sin formación/competencias necesarias Desmesurada medicalización a usuarios/as de Salud Mental Nivel precario de competencias clínicas, considerando perfil de usuarios

Tabla 3: Construcción de categorías y subcategorías conceptuales (en cursiva los conflictos éticos percibidos como críticos). 
nes en las cuales no tienes respuesta clara... no sabes ciertamente como actuar"; I3-R2; "(...) hechos con elementos contrapuestos para intervenir o ejecutar alguna acción", I7-R1: "Disyuntivas valóricas en el ámbito prefesional", I8-R1; "Algo que transgrede mis principios y valores, como persona y profesional', I24-R1. Solo un informante no detectó problemas éticos asociados a su ejercicio profesional. Lo explicó refiriendo que sí aprecia múltiples conflictos, pero los entiende como propios del trabajo, la naturaleza de las atenciones y el contexto, pero no los perfila como éticos, ya que a éstos los asimila estrictamente asociados a hechos como conflictos de interés, algo que señala ausente en su experiencia.

La categorización permitió clasificar los conflictos en tres planos diferentes, los que a su vez se subcategorizaron, como se describe en la tabla 3.

\section{Discusión}

Para fines de síntesis y encuadre del texto, se privilegiará la revisión por representatividad de los cinco conflictos éticos que fueron considerados como críticos por al menos dos prefesionales, según la categoría correspondiente: Atención a compañeros de labores y/o a sus familiares; Extenso periodo intercontrol de usuarios/as; Sobredemanda de funciones como impedimento de calidad en cometidos; Obligatoriedad de denunciar y su relación costo/efectividad, y Limitada resolutividad del nivel de atención.

\section{Atención a consultantes}

El conflicto sobre la obligatoriedad de denunciar y su relación costolefectividad da cuenta de las aprensiones surgidas ante distintos casos, en los cuales, por su naturaleza y características, los profesionales deben hacer la denuncia respectiva según las normativas legales vigentes. Siendo los ejemplificados en su mayoría relacionados con vulneraciones a los derechos de infantes, no se duda sobre la acción de denunciar los acontecimientos en las instancias respectivas, sino que el cuestionamiento surge sobre los efectos e implicancias derivadas de cada procedimiento y posterior proceso a cursar. "Porque uno piensa, realmente ¿qué va a pasar con este niño o niña... o con esta madre? Denunciar igual es complejo, y tú tienes el conflicto de que sabes que lo tienes que hacer, para también sabes que tal vez no es lo mejor... por las consecuencias" Informante 9; Respuesta 7.

Los reparos y objeciones en su mayoría pormenorizan las experiencias de cada profesional ante las situaciones de que los menores son separados de sus familias y llevados a centros de acogida y residenciales transitorios o definitivos, o en otros casos entregados para el cuidado o tutela de familiares, escenarios que en ocasiones han repercutido en transgresiones y/o negligencias similares e incluso peores a las iniciales.

Que se pueda resguardar y asegurar los derechos a cuidados infantiles mínimos es una tarea custodiada por el Estado y designada en cada servicio, lo que supera el alcance de la labor del profesional de salud mental. Sin embargo, los informantes destacan que el inicio formal de estas causales, así como sus repercusiones, es cuando se enteran de la información y cumplen con la exigencia propia del funcionario público de poner en conocimiento de tales hechos a las autoridades correspondientes.

Dejan claro que en ningún caso el actuar es pensado a modo de ocultar y/o silenciar lo descubierto, pero que sí es fundamental analizar la casuística de cada situación y diferenciar de manera clara y oportuna aquellos casos que revisten o no carácter de delito, actos que van más allá del límite interpretativo y prudencial del profesional de APS(10), e involucran a la red socioinstitucional pública.

El conflicto acentúa la precariedad con que se concibe a la red de derivación y apoyo formal existente y las barreras burocráticas del sistema judicial, y cuestiona el rol de los actores involucrados en toda esta trama, comenzando por los profesionales mismos. Apelando a contar con los mecanismos y herramientas necesarias para la efectiva y adecuada resolución, ante hechos como este convendría hacer una lectura crítica desde la interacción de principios como la no maleficencia-beneficencia $(11,12)$, y adquiriría relevancia el incorporar conceptos desde el principio de protección $(13,14)$, lo que podría facilitar la discusión y argumentación en este complejo escenario. 
Conociendo de primera fuente el tipo de intervenciones que se enmarcan en este nivel de atención, su correspondiente sentido de pertenencia y la demanda por la eficiente utilización de los elementos disponibles, los informantes caracterizan la limitada resolutividad de nivel de atención a modo de exponer lo acotado de las acciones que muchas veces pueden ejecutar, y cómo éstas, a su vez, están condicionadas a otro tipo de recursos restringidos(15); por ejemplo infraestructura, movilización y, por supuesto, el humano. "(...) porque personalmente me ha tocado ver el impacto que tiene en los usuarios... cómo no se les resuelven los problemas, cómo los patean de un lado a otro, se siguen retirando... asi se minimiza cualquier posibilidad de intervención..." I17-R6; "Se intenta hacer lo mejor con lo que se tiene, y es indudable que se ha mejorado... pero siempre se puede hacer un poquito mejor, ¿o no? Hay varias cosas que mejorar, sobre todo si podemos contar con más recurso humano", I24-R6.

En estos profesionales suele recaer una función reguladora, ya que asumen la detección de trastornos mentales y la asistencia a problemas psicosociales que no requieren de tratamiento especializado, determinando la conveniencia o no de la derivación a nivel secundario, el cual ya se encuentra sobredemandado. Por una parte, se evidencia que este patrón ha mostrado limitaciones importantes, debido a escasez de recursos destinados a la APS, y, por otra, que la asistencia de carácter psicológico ha sido desplazada e ignorada en términos de planificación estratégica y desarrollo de servicios, lo que ha conducido a una subutilización, subfinanciación y subdesarrollo de los tratamientos psicológicos en la asistencia sanitaria(16), que finalmente atenta contra la adecuada gestión y repercute en la calidad de la atención misma.

\section{Relación entre colegas y con otros profesionales de la salud}

La atención a compañeros de labores y/o a sus familiares es el conflicto ético de mayor número de menciones espontáneas, así como también el que la mayoría de los entrevistados coincidió en denominarlo crítico (tabla 2). Este se relaciona de manera intrínseca con la percepción y sentido del deber profesional, al sopesar el posible impacto presente y posterior de transgredir límites profesionales, de compañerismo y amistad eventuales que estén involucrados: "Es que implica meterte en una relación que va más allá de tu rol como psicólogo, porque aunque no quieras se mezcla la amistad que puede haber, con lo laboral... y eso enreda las cosas... quedas en una posición compleja" -I 11 - R 5-; "Es más difícil esclarecer los limites (...) los funcionarios tienden a confundir los limites profesionales con los personales", I18-R 5.

El que los profesionales debiesen realizar este tipo de atenciones denota lo limitado de las redes asistenciales de salud con las que se cuenta, ante posibles derivaciones y acogidas de casos de tal naturaleza. No tan solo tensiona las relaciones existentes en el seno de la institución de salud, sino que compromete la base necesaria de confianza, intimidad y confidencialidad que se debe asegurar en la atención $(17,18)$, ante todo en la de salud mental.

Asimismo, cada informante fue enfático al señalar que, como este hecho representaba especial inconveniente, es uno de los que ya han tenido repuestas satisfactorias en cuanto a la proyección de vías resolutivas. En una labor conjunta con los entes coordinadores, se han puesto en práctica distintas alternativas conducentes a procedimientos locales para resolver situaciones concretas, experiencias que a su vez han servido como buenas prácticas replicables en otros establecimientos de la red de APS.

\section{Contexto de trabajo en salud pública}

El extenso periodo intercontrol de usuarios/as refiere principalmente al ideal de que la periodicidad de las atenciones no sobrepase la frecuencia semanal y/o quincenal, dependiendo de las características de cada caso. "Es algo que va más allá de lo profesional, eh... tiene que ver con la falta de recursos humanos... ¿cierto? ; $Y$ cómo se supone que vas a ayudar a la salud mental así?', I 4 - R4. Algunos informantes referían que los periodos entre uno y otro control incluso podrían llegar a los cuarenta y cinco días, coincidiendo en cómo afectaba distanciar evoluciones precarizando cualquier resultado terapéutico, haciéndolos no efectivos.

Este conflicto devela la sobredemanda de las aten- 
ciones de salud mental y la insuficiencia de los recursos disponibles, así como la baja cantidad de profesionales per cápita. A la vez expondría una falla en la pesquisa y/o detección de los problemas de salud mental, y la posible incorrecta derivación hacia el programa, enviando casos que no sean del perfil requerido, de sintomatología no significativa o que manifiesten otro tipo de problemáticas ajenas a las estrictamente enfocadas en el área, lo que atesta las agendas y genera el progresivo aumento en las listas de espera.

Desenmascara lo necesario de revisar los criterios de derivación para diferenciar a los usuarios/as reales del programa, según las directrices determinadas, así como enfatizar la funcionalidad de los procedimientos implementados(19). Ante estas circunstancias, puede ser provechoso promover una "cultura de la psicología en atención primaria”, en el sentido de pasar de un modelo de vulnerabilidad (dolencia e indolencia) a uno de capacidad de recuperación (potenciación de todas las capacidades de las personas; gestión de la facultad de agencia individual y comunitaria)(20), de manera que los usuarios/as hagan un uso más racional de los servicios.

Por último, la sobredemanda de funciones como impedimento de calidad en cometidos pesquisada en este estudio se erige como el hecho distintivo y de los más representativos de la APS. Es frecuente que un variado número de obligaciones recaigan en un mismo profesional, si se considera que la cantidad de recurso humano es baja para la creciente proporción de actividades. "(...) sin duda que altera el trabajo... que te interrumpan a cada rato... tu te esfuerzas, pero afecta la calidad de lo que haces igual...", I 16 - R6; "Es recurrente esto, pasa a diario... o casi todos los días. Al final terminas cansado con esto... pasa y pasa; y tú ahi dale que dale... Al final afecta la calidad de lo que haces, el trabajo y el producto que entregas", I 23 - R 6.

Los informantes reconocen avances en la incorporación de recurso humano, pero la calidad cada vez más alta exigida complejiza los procesos(21), genera nuevas demandas que sobrepasan las propias atenciones circunscritas al área de la salud mental(22) y redistribuye funciones, lo cual vuelve a multiplicar tareas.
Cualquier asignación de tiempo tropieza con la priorización y, en modelos basados en metas, el énfasis desmedido del sistema público en evaluar programas por el número de personas atendidas y/o prestaciones entregadas también atenta contra la calidad de atención(23). En situaciones como las descritas, aunque un profesional esté capacitado para realizar las tareas encomendadas, el nivel de sobrecarga y la falta de tiempo dificultarían realizarlas adecuadamente(24). Se puede suponer que estas situaciones no tan solo afecten el nivel de la calidad de las prestaciones, sino que además incrementen el nivel de desgaste emocional y el estrés de los profesionales.

\section{Conclusiones}

De los distintos ámbitos humanos, la ética es imperativa cuando de tratar a personas se refiere, más aún aquellas que de una manera u otra se entregan al cuidado de otro(25). El estudio permitió acceder, en líneas generales, a las problemáticas detectadas por los informantes, coincidiendo estos en que varios de estos conflictos han permanecido naturalizados y luego normalizados en el tiempo.

Surge la necesidad de identificar y revisar quiénes son los actores involucrados en la materia; analizar el sujeto de la acción (quién forma parte en el problema y quién tiene que colaborar en la resolución de éste), la actuación específica (cómo se adapta a los principios éticos) y las consecuencias que puede tener la ejecución de una determinada actuación concreta(26), para visibilizar los cuestionamientos levantados y poder trazar una línea de base que establecezca mínimos permitidos de común acuerdo. Tales resoluciones aportarían a desatar nudos clave en lo que representa la multidimensionalidad del trabajo que debe articular la psicología en APS.

Dada la naturaleza de las atenciones en salud mental, se vislumbra que los conflictos nos cesarán: a medida que se encuentran vías resolutivas para unos, otros se irán generando, como parte de la diversidad y complejización de demandas. Por lo mismo, solo profesionales debidamente preparados y atentos a las distintas necesidades pueden ser garantes de intervenciones de calidad y excelencia requerida. 


\section{Conflicto de intereses}

Tres de los veinticinco entrevistados mantienen relación de amistad desde antes del estudio con el autor. Se resguardó cualquier sesgo aplicando exactamente la misma pauta de preguntas a todos/as y cada uno/a de los/as participantes de la investigación.

\section{Agradecimientos}

La investigación no hubiese sido posible sin la cooperación de la Coordinadora de Salud Mental del Departamento de Salud Comunal de Talca, y la colaboración de cada uno/a de los y las psicólogos/as que accedieron a las entrevistas, a quiénes se agradece su participación.

\section{Referencias}

1. Organización Mundial de la Salud. Información sobre la salud en el mundo 2008. La atención primaria de salud. Más necesaria que nunca [Internet]. Ginebra: OMS; 2008 Disponible en: http://bit.ly/1TfSvkm

2. Gracia D. Bioética clínica. Bogotá: Editorial El Búho; 2001.

3. Zurro M, Cano J, Gené J. Atención Primaria. Principios, Organización y Métodos de Medicina en Familia. España: Elsevier; 2014.

4. Bedregal P. Ética en las organizaciones de salud. En: Beca J, Astete C, (eds.). Bioética clínica. Santiago: Mediterráneo; 2012: 513-524.

5. Monsó C, Sales P, Loncán P, Mateos A, Talavera M, Zapater F, Fernández M, Gracia E, Carrera S, Cladellas E, Velasco R, Tias A, Beunza P. Estresores éticos y atención sanitaria. Análisis de estresores éticos en el ámbito del CèAVOC (Comité de Ética Asistencial del Vallés Oriental Central). Revista Bioética y Derecho 2017; 41: 107-119. DOI: http://dx.doi. org/10.1344/rbd2017.41.18135

6. Vidal M, Pérez A. Formación en Valores. Conceptos éticos y tecnológicos, métodos y estrategias. Educación Médica Superior 2016; 30 (4) Disponible en http://ems.sld.cu/index.php/ems/article/view/881/448

7. Gracia D. Fundamentando la Ética Clínica. En: Beca J, Astete C, (eds.). Bioética clínica. Santiago: Mediterráneo; 2012: 32-49.

8. Moncada, S. Psicología y Atención Primaria de Salud: Una propuesta de competencias. Revista Chilena de Salud Pública 2015; 19(3): 293-304. DOI: 10.5354/0719-5281.2015.37646

9. Hermosilla A, Liberatore G, Losada M, Della Savia P, Zanatta A. Dilemas éticos en el ejercicio de la psicología: resultados de una investigación. Fundamentos en Humanidades 2006; 7(1-2): 91-106

10. Echeburúa, E. El secreto profesional en la práctica de la Psicología Forense: alcances y límites de la confidencialidad. Análisis y Modificación de Conducta 2002; 28(120): 485-501.

11. Beauchamp T, Childress J. Principios de ética biomédica. Barcelona: Masson; 1999.

12. Siurana J. Los principios de la bioética y el surgimiento de una bioética intercultural. Veritas 2010; (22):121-157. DOI: https://dx.doi.org/10.4067/S0718-92732010000100006

13. Schramm F, Kottow M. Principios bioéticos en salud pública: limitaciones y propuestas. Cadernos de Saúde Pública 2001; 17(4): 949-956.

14. Pérez-Tamayo R. Ética médica, salud y protección social. Salud Pública de México 2005; 47(3): 245-251.

15. Bedregal P, Leal V, Lavados M, Lafuente M, Chomalí F, Ardiles J, Martínez S, Boeusseu M. Asignación de recursos en salud en Chile: Consideraciones éticas en la toma de decisión. Revista Médica de Chile 2002; 130(2): 181-190.

16. Pastor J. El Psicólogo en Atención Primaria: Un debate necesario en el Sistema Nacional de Salud. Papeles del Psicólogo 2008; 29(3): 271-290.

17. Judez J, Nicolás P, Delgado T, Hernando P, Zarco J, Granilleras S. La confidencialidad en la práctica clínica: historia clínica y gestión de la información. Medicina Clinica (Barcelona) 2002; 118(1): 18-37.

18. Judez J. Intimidad, privacidad, confidencialidad y secreto en la práctica clínica. En: Beca J, Astete C, (eds.). Bioética clínica. Santiago: Mediterráneo; 2012: 160-177.

19. Domínguez O, Manrique C. Bioética y calidad en la atención de primer contacto y de salud pública. Acta Bioethica 2011; 17(1): 31-36. DOI: http://dx.doi.org/10.4067/S1726-569X2011000100004

20. Pérez M, Fernández J. Más allá de la salud mental: la psicología en atención primaria. Papeles del Psicólogo 2008; 29(3): 251-270.

21. Oyuela M. La importancia de la reconciliación entre Eficiencia y Equidad, para los sistemas de salud, desde una perspectiva ética. Revista Latinoamericana de Bioética 2010; 10(2): 100-107. DOI: https://doi.org/10.18359/rlbi.1009 
Conflictos éticos detectados por psicólogos/as de la atención primaria de salud - Manuel Pérez Ayala

22. Scharager J, Molina M. El trabajo de los psicólogos en los centros de atención primaria del sistema público de salud en Chile. Revista Panamericana de Salud Pública 2007; 22(3): 149-159. DOI: 10.1590/S1020-4989200700080000

23. Minoletti A, Rojas G, Horvitz-Lennon M. Salud Mental en Atención Primaria en Chile. Cadernos de Saúde Coletiva 2012; 20(4): 440-447. DOI: 10.1590/S1414-462X2012000400006

24. Moreno M. El tratamiento de problemas psicológicos y de salud mental en Atención Primaria. Salud Mental 2012; 35(4): 315-328. Disponible en http://www.redalyc.org/articulo.oa?id=58224380007

25. Dörr O. Ética y Psiquiatría. Aspectos de la praxis cotidiana. Revista Colombiana de Psiquiatría 2002; 21(1): 27-48.

26. García M. Análisis de dilemas éticos en atención primaria. Medicina de Familia (Andalucía) 2000; 1: 89-93.

Recibido: 6 de febrero de 2019

Aceptado: 19 de febrero de 2019 